\title{
Psychothérapies et approches plurielles de leur évaluation
}

\section{Psychotherapies and diverse approaches of their evaluation}

\author{
G. Fischman \\ (C) Springer-Verlag France 2010
}

Je suis reconnaissant au comité de rédaction de la revue $P S N$, de l'honneur de me permettre d'aborder dans cet éditorial l'importance des psychothérapies dans la pratique des psychiatres. Les psychothérapies constituent, aujourd'hui, une pratique sociale complexe qui engage notre perception de l'humain. Les professionnels sont actuellement au centre d'une demande d'aide psychologique, certes des soins, mais aussi d'un questionnement profond sur les moyens de répondre aux attentes d'épanouissement personnel, relationnel et affectif. Ces exigences imbriquées et, naturellement, indissociables comportent, quand elles s'adressent au clinicien, un agrégat d'attentes fusionnées dont il est ardu de faire la distinction, d'une part, entre la détermination diagnostique d'une symptomatologie caractérisée et, d'autre part, les difficultés existentielles diverses. Autrement dit, les attentes avancées par les demandeurs d'aide psychothérapique concernent tout autant la disparition des symptômes de dysfonctionnement psychique, la réduction de l'anxiété éprouvée lors des situations vécues que la dimension humaine inévaluable qui dépasse les limites de la technicité pour accéder à une perception de la vie affective et sociale, avec ses exigences et ses difficultés.

Ces besoins sociaux se sont massifiés progressivement depuis quelques décennies, pour se développer tous azimuts au-delà des sentiers existants à l'intérieur du champ des spécialistes de la psychopathologie, psychiatres et psychologues [1]. Aujourd'hui, le coaching, les thérapies « alternatives » innombrables, les stages intensifs de développement personnel, les approches biocorporelles les plus hétéroclites se sont adjoints aux approches psychothérapies classiques. L'extension grandissante de ces attentes a produit une nouvelle offre d'aide psychologique par des nouveaux acteurs sociaux revendiquant un savoir-faire d'autant plus spécifique et « technique » que leur formation psychopathologique générale est

G. Fischman $(\bowtie)$

Service hospitalo-universitaire de santé mentale et de thérapeutique (Pr J.-P. Olié),

centre hospitalier Sainte-Anne,

7, rue Cabanis, F-75014 Paris, France

e-mail : g.fischman@ch-sainte-anne.fr manquante. La réglementation de ce champ de professionnels se réclamant compétents en psychothérapie s'est mise, en France, laborieusement en cours depuis un lustre. Ces phénomènes de civilisation interpellent avec raison les soignants sur la définition des réponses professionnelles apportées et leur rôle dans la société contemporaine. En effet, l'ouverture de la souffrance psychologique au marché de l'offre des soins et l'émergence d'une nouvelle professionnalisation de psychothérapeute en cours ont mis la question des psychothérapies au-devant des préoccupations de la psychiatrie.

Cette expansion progressive du champ d'application des psychothérapies, la prolifération des techniques et le développement des écoles diverses ont généré la nécessité de clarifier les indications, d'évaluer les résultats ainsi que de comprendre les processus spécifiques aux différentes techniques et leurs facteurs communs d'actions [12]. Aux questionnements cliniques des praticiens se sont associées, aux États-Unis, les contraintes imposées par le système de remboursement des soins américain. Ces organismes privés de financement des frais de santé, plus intéressés à réduire les coûts qu'à établir la qualité des soins au long cours, ont eu une incidence majeure dans le développement de la recherche empirique, afin d'évaluer l'efficacité des psychothérapies.

En France, dans le cadre de la réglementation publique des psychothérapies, initiée dans les années 2000, quelques voix se sont manifestées aussitôt pour soulever « l'exigence éthique » de procéder à une évaluation de leur efficacité supposée. Et pour certains, il serait devenu même urgent et donc impératif de connaître objectivement les effets comparatifs des techniques différentes - notamment psychanalytiques et cognitivocomportementales - alors qu'il s'agissait sur le fond d'élaborer une politique dans le champ social de l'aide psychologique. De fait, un véritable défi a ainsi été lancé aux praticiens. C'est dans ce contexte qu'a été commandé à l'Inserm un rapport d'expertise sur l'efficacité comparée des approches psychothérapiques, sous l'égide d'une démarche scientifique calquée sur la recherche biomédicale, et selon les principes en vigueur de l'evidence-based medicine (EBM). Parue début 2004 en France, l'analyse bibliographique de l'Inserm a été à l'origine de vifs débats 
qui ont envahi les médias. À l'origine d'une des plus grandes polémiques entre les représentants des courants psychothérapiques dans laquelle la psychiatrie a été impliquée, ce rapport, controversé dans ses principes et ses conclusions, a provoqué un clivage jusqu'alors inédit entre pratiques différentes, mises ainsi en concurrence dans l'espace sanitaire, sociale et universitaire. Derrière une rhétorique scientifique limitée à la culture médicale, réduisant la problématique clinique à une liste de troubles et uniformisant les approches pour le faire rentrer dans une démarche de satisfaction, en lien avec la réduction des symptômes, le rapport de l'Inserm a rempli la malheureuse fonction de fer de lance de la dite " guerre des psy », c'est-à-dire la confrontation des cliniciens d'orientation psychodynamique et ceux d'orientation cognitivocomportementale autour des divergences des conceptions psychanalytiques et cognitivistes du sujet. C'est dans ce contexte historique qu'a fait irruption, de manière d'autant plus passionnelle, la recherche en psychothérapie.

Il nous a paru nécessaire, après cette période tumultueuse des confrontations polémiques, d'apporter des réponses pertinentes au sujet de l'évaluation des psychothérapies en général et des psychothérapies psychanalytiques en particulier. Cette nécessité a motivé l'élaboration d'un ouvrage collectif réunissant des chercheurs et des cliniciens autour des problématiques méthodologiques et épistémologiques soulevées par cette évaluation [3].

Nous sommes certains que cet ouvrage, conçu dans un esprit pluriel d'analyse et de confrontation épistémologiques, offre un outil de réflexion approfondie et une référence incontournable pour tout positionnement concernant ce domaine de recherches. Dans ce sens, l'Association française de psychiatrie, témoignant de tout l'intérêt que notre corps professionnel prête à la dimension psychothérapique, a organisé un colloque à Paris, en octobre 2009, fondé sur les contributions de la plupart des auteurs de ce collectif devenu ainsi l'axe de réflexion fondamentale des professionnels des psychothérapies en France.

\section{Évaluation des psychothérapies : histoire et controverses}

Quelle thérapie, pour quelle pathologie et pour quel patient ? Voilà les questions principales auxquelles certains espèrent trouver des réponses univoques. Ces interrogations sont évidemment légitimes, car elles concernent les attentes des patients tout autant que les indications posées et les objectifs fixés par les professionnels dans l'anticipation de leurs effets. Néanmoins, le souci de l'adaptation thérapeutique à chaque patient n'est pas une préoccupation à la mode du jour, ces questionnements ont marqué, dès l'origine, l'histoire des approches psychothérapiques. Les réponses appor- tées à cette problématique ont toujours buté sur les difficultés de l'objectivation et de la mesure des réalités psychiques. Mais les normes de l'évaluation ont beaucoup évolué, et un fort mouvement d'inspiration médicoscientifique est devenu dominant sur le plan de la recherche.

Ainsi, la recherche sur les psychothérapies a déjà une histoire de plus d'un demi-siècle, et elle est extraordinairement dense. Ces recherches que l'on définira d'empiriques, selon la dénomination internationale en usage, sont définies par l'utilisation des questionnaires standardisés aptes à l'analyse statistique. Pendant une première période, celles-ci étaient focalisées essentiellement sur la réduction des symptômes selon les pathologies, les données démographiques et épidémiologiques [5].

De manière classique, on distinguera ainsi les études sur :

- le processus psychothérapique ;

- les résultats cliniques au sein desquels on a institué la différenciation formelle entre l'efficacité potentielle dans le cadre des protocoles expérimentaux (efficacy) et l'efficacité réelle avec des résultats obtenus par le biais des protocoles « naturalistes » (effectiveness);

- les relations causales entre le processus et les résultats.

La prolifération des études de qualité inégale a conduit à l'établissement de normes de qualité selon les critères calqués de l'EBM, témoignant d'une rigueur méthodologique croissante. Ainsi se sont imposés pendant une décennie, les principes de l'empirically-supported psychotherapy ou " psychothérapie empiriquement fondée ", d'après lesquels seules les psychothérapies (cogniticocomportementales) ayant prouvé leur efficacité par des études contrôlées mériteraient ce label de qualité présumée.

Le paradigme de cette méthodologie est représenté par les études contrôlées randomisées (ECR), modèle issu des essais pharmacothérapeutiques, assurant la fidélité et la validité des protocoles répondant aux exigences de l'objectivité. Ces études nécessitent de répondre aux exigences suivantes : un protocole intégrant des critères d'inclusion sévères, la randomisation des traitements, la constitution de groupes témoins, l'utilisation d'un manuel d'intervention, les métaanalyses des études primaires.

Néanmoins, de multiples objections ont été soulevées à propos de la notion des "psychothérapies empiriquement fondées ", et des contestations virulentes se sont manifestées à l'établissement des «listes de psychothérapies empiriquement prouvées » qui en exprimaient le corollaire [6]. Ce type d'essais correspond-t-il aux conditions des pratiques psychothérapiques réelles ? Sur le plan méthodologique, de nombreux chercheurs ont reconnu que l'attribution de valeur probante accordée aux ECR n'est pas ici justifiée. En effet, le souci de préserver la validité interne, représenté par l'utilisation des critères sévères d'inclusion des sujets, la prise en compte de variables symptomatiques limitées et l'exclusion 
de polypathologies conduisent à des études qui n'intègrent pas le caractère pluridimensionnel et interactif des demandes d'aide psychothérapique et compromettent paradoxalement la validité externe de leurs résultats. Autrement dit, la cohorte de patients inclus dans les études n'est plus représentative des patients de la clinique réelle.

Ainsi, par la recherche des preuves scientifiques tributaires de la logique de l'EBM, ces critères se sont transformés en une nouvelle orthodoxie méthodologique, en raison du rôle privilégié accordé aux ECR et aux méta-analyses, brisant l'équilibre entre les résultats obtenus par des protocoles supposés de « qualité expérimentale » et des études « naturalistes " plus proches de la réalité du travail réel des cliniciens. Cette "méthodolatrie » ne pouvait que creuser le fossé existant entre les chercheurs et les praticiens de référence psychodynamique.

En conséquence, il est devenu aussi indispensable d'évaluer avant tout les critères de ces évaluations. Que souhaitet-on réellement évaluer du travail psychothérapique?

- Serait-ce la compréhension des processus du changement psychique?

- S'agit-il de la détermination de l'efficacité d'une approche psychothérapique donnée pour la réduction des symptômes manifestes ou bien de comprendre les effets sur la mobilisation des ressources dynamiques de la personnalité ?

- Plus précisément, est-il certain qu'une psychothérapie courte sera plus efficace sur les symptômes et plus économiquement rentable qu'un travail long axé sur la dynamique de la personnalité ?

- S'agit-il de faire des corrélations, à plus ou moins courte échéance, sur le rapport des coûts/bénéfice dans une logique de maitrise des dépenses de santé ?

- Pourra-t-on établir, de manière statistique, quelle thérapie sera plus adaptée à chaque tableau psychopathologique et en définir des normes techniques auxquelles il faudrait se conformer?

\section{Nécessité de l'analyse épistémologique}

L'attente de réduire le niveau le plus complexe de l'action psychothérapique au domaine des études réalisées dans le champ biomédical restera problématique et équivoque. En effet, la recherche en psychopathologie quantitative a développé des outils standardisés de mesure, appliqués à la clinique psychiatrique et à la thérapeutique psychopharmacologique.

Pourtant, une interrogation s'impose de manière récurrente : est-il pertinent d'étendre les mêmes principes et les mêmes outils méthodologiques, adaptés à l'exploration des symptômes patents, au niveau où se produit l'émergence la plus subtile de la subjectivité, et de surcroît, lors des interactions créées dans un champ thérapeutique duel et intersub- jectif ? Est-il possible d'identifier un sujet, considéré dans sa complexité et dans sa singularité, à une cohorte/moyenne, cette figure déduite des procédés du calcul statistique, et de négliger, par la suite, les effets de cette normalisation sur les individus ? Des réserves avaient déjà été élaborées à ce sujet autour des notions de normalité et de normativité. Le risque existe alors de s'orienter vers un formalisme pseudoscientifique qui instrumentalise les méthodes des sciences, masquant sous l'objectivité des protocoles une conception qui néglige la singularité des sujets [8].

\section{Psychanalyse et recherche}

Les logiques psychanalytiques sont la résultante d'une approche thérapeutique complexe. Même jugées hétéroclites, ces approches rendent compte de réalités subjectives multiples, et leur validation clinique reste conditionnée à l'évaluation renouvelée lors du travail des cas singuliers. Cela ne pouvait que rendre les praticiens du champ psychodynamique pour le moins sceptiques vis-à-vis de toute démarche méthodologique purement quantitative. Il est clair, dans la position de ces cliniciens, que le processus d'investigation heuristique de la clinique ne doit pas être réduit à la méthodologie de la recherche empirique standardisée.

En effet, ceux qui défendent une logique de validation clinique des effets thérapeutiques considèrent les recherches empiriques comme une impasse méthodologique apportant des résultats négligeables, au regard de la sophistication des techniques statistiques mises en œuvre. La validation ne peut être issue que d'un modèle clinique avec des logiques propres aux sciences humaines : une théorie de la justification fondée sur la méthode hypothéticodéductive, attachée aux études de cas singuliers et orientée vers les recherches de type exclusivement qualitatif. Pourtant, cette rationalité « constructiviste » a donné lieu à des critiques acerbes en tant que procédé de validation des données psychanalytiques. L'argument essentiel est fondé précisément sur le caractère éminemment subjectif de cette " autoréflexion 》 imprégnée des mécanismes suggestifs. Pourtant, au-delà des certitudes des praticiens du champ psychanalytique, certains attendent des vérifications objectives des résultats par des études « scientifiquement irréprochables ». Ces expectatives seraient certes bien fondées s'il ne s'agissait pas, dans ces recherches, d'évaluer un objet aussi singulier que la subjectivité des sujets dont la complexité rend ces attentes souvent illusoires et décevantes.

\section{Modèles standardisés classiques}

Ainsi, les praticiens se référant à la psychanalyse se trouveraient-ils en état de siège épistémologique, cernés, d'un côté, par un pragmatisme positiviste et, de l'autre, par un idéal herméneutique retranché derrière la forteresse d'une 
subjectivité insondable? Or, une autre perspective s'offre désormais aux cliniciens : au lieu de se cantonner à la seule défense d'un sujet transcendant il est désormais, méthodologiquement possible d'affronter les paradigmes scientifiques sur leur propre terrain, par la défense de la rationalité qui leur est propre, atteignant le niveau le plus complexe de l'humain, sa singularité.

De nombreux praticiens considèrent que les données psychanalytiques devraient être abordées aussi par des méthodes expérimentales ${ }^{1}$ et soulignent l'intérêt de réaliser des recherches répondant aux mêmes critères établis pour les recherches en psychothérapie. Ceux-ci soutiennent qu'il existe un potentiel de vérification empirique, d'où la nécessité de renouer avec l'essor des disciplines scientifiques connexes et d'apporter les preuves de l'efficacité réelle des thérapeutiques psychanalytiques, selon les critères d'évaluation en vigueur.

Pour cette école de pensée, développer ce type de recherches permettra de valider, face à la communauté scientifique, les résultats des actions thérapeutiques de la psychanalyse. Il s'agirait, selon ces auteurs, de ne pas se priver de l'application des critères objectifs quantifiables, en interaction avec la pratique analytique.

En tout cas, toute recherche dans ce domaine nécessite un abord proprement psychanalytique [11]. Un bref rappel de ces enjeux nous paraît un préalable à ces questionnements d'ordre méthodologique :

- quelle est la définition de la technique à évaluer ?

- Quelles seraient les mesures adéquates d'évaluation des effets?

- À quelle distance du travail psychanalytique faut-il en évaluer les effets durables?

- Si ce n'est pas l'effacement du symptôme, mais la qualité de vie qui doit être évaluée : est-elle évaluable par des questionnaires individuels ou bien faut-il aussi la mettre en évidence par des indicateurs indirects?

- Les évaluations seraient-elles mieux accomplies par des mesures concernant le sujet individuellement ou doiventelles être ouvertes également au champ extraclinique, sanitaire et social ? [9] ;

- en ce qui concerne les données spécifiquement psychanalytiques, s'agira-t-il de relever la dynamique des défenses, de décrire la dyade transférentielle ou d'évaluer la qualité des relations d'objet?

\footnotetext{
${ }^{1}$ Plusieurs études sont devenues classiques : celles de Coriat en 1917, de Knight en 1941 ; celles de l'institut de Boston; le projet du centre de psychanalyse de Columbia ; celles de l'institut de New York, de la clinique Menninger ; la recension du centre Anna-Freud ; l'étude de l'Association psychanalytique allemande (Leuzinger-Bohleber) ; l'étude suédoise (Sandell).
}

En tout cas, les évaluations à court terme, limitées à la réduction des symptômes, n'ont pas de validité suffisante pour mesurer l'action thérapeutique durable [11]. Les résultats seront mieux évalués avec la prise en compte de l'évolution des capacités psychologiques globales du patient, de la qualité des relations interpersonnelles et de l'évolution de la dynamique propre de la personnalité.

\section{Modèles empiriques idiographiques et protocoles de cas singuliers}

Même si la méthodologie de groupe est nécessaire pour la recherche, la constitution des groupes homogènes est, par définition, insensible aux exigences de l'individualisation des pratiques cliniques, indéfectiblement concentrées sur la notion du cas singulier. En conséquence, nous comprendrons l'intérêt de bien différencier les recherches psychothérapiques focalisées sur le traitement, construites sur la base des moyennes de groupes homogènes, et les recherches psychothérapiques focalisées sur le patient, centrées sur des cas individuels ou sur la dyade patient-thérapeute, qui comportent une exploration holistique des cas singuliers.

Depuis une quinzaine d'années, un nouveau paradigme devient déterminant pour le développement d'une alternative aux recherches empiriques sur les psychothérapies en général et sur celles de référence psychanalytique en particulier : l'étude de cas singulier ou single-case design. Actualisant la valeur de l'étude approfondie classique d'un seul sujet, le protocole de l'étude de cas singulier, véritable troisième voie des recherches, est de nature standardisée selon le principe de l'objectivité expérimentale. L'analyse d'un seul cas permettra d'inférer un nombre important de constats, car l'individu représente ici la totalité de la "population » étudiée. À la différence d'un protocole extensif concernant un groupe, dans un protocole intensif de cas singulier on cherche l'approfondissement systématique d'un seul cas clinique.

Les références spécifiquement psychodynamiques de ces outils standardisés les rendent adaptés à l'approche des données psychanalytiques et laissent espérer une nouvelle génération de recherches, à la fois plus proches du vécu réel des patients ainsi que des convictions véritables des praticiens ${ }^{2}$. C'est la seule voie de recherche empirique, pour ceux qui s'engageraient à la difficile réalisation des études standardisées, qui pourra s'approcher de l'essence du processus psychothérapique et des effets de la thérapeutique psychanalytique.

\footnotetext{
${ }^{2}$ Citons parmi les échelles qui nous semblent les plus représentatives : a) processus psychothérapique Q-Sort de Enrico Jones ; b) la SWAP 2000 de Shedler et Westen et al. ; c) protocole herméneutique d'efficacité de cas singuliers de Robert Elliot ; d) formulation idiographique des conflits de Christopher Perry ; e) analyse configurationnelle d'Horowitz et Eells.
} 


\section{Enjeux anthropologiques de l'évaluation : l'idéal de la gouvernance}

Enfin, notre propos introductif manquerait d'une analyse globale des enjeux si nous faisions l'impasse sur la dimension sociale et politique que comporte l'extension des évaluations quantitatives dans beaucoup de secteurs de notre culture. En effet, nous ne sommes pas les seuls professionnels ayant été confrontés, ces dernières années, aux tumultueuses avancées des évaluations des acteurs du champ social. Celles-ci semblent devenir un principe de contrôle gestionnaire, voire un dispositif gouvernemental assumé comme une évidence de «bon sens », fondé sur des critères supposés objectifs qu'il serait regrettable de contester. Ce nouveau langage quantitatif crée un style de « gouvernance » de tous les secteurs des interventions sur l'humain, auquel on ne pourrait plus déroger (école, hôpital, enseignement et recherche en sciences humaines, psychothérapies, etc.) [13].

Certes, l'objectif est de contrôler ou de justifier la rentabilité d'une prestation, de développer la mesure de la satisfaction des usagers d'un service, d'identifier les points d'amélioration possible d'un travail et donc de corriger ses pratiques de façon à en accroître l'efficacité. Néanmoins, les buts de l'évaluation, selon les acteurs impliqués, peuvent être opposés et contradictoires ; les résultats de l'évaluation servent de moyen de contester le pouvoir de l'autre. " $\mathrm{Si}$ l'évaluation est une arme au service de la controverse dit un spécialiste du contrôle des affaires sociales — alors nombreux seront ceux qui s'ingénieront à l'édulcorer, à l'empêcher ou à la rendre sans effet. Si elle est un moyen de forger un nouvel équilibre entre des intérêts différents et reconnus dans leurs différences, alors elle peut être un moyen de progresser collectivement » [10].

Au temps de l'homme numérique et de la mathématisation $d u$ monde, l'évaluation quantitative s'avère être la matrice d'une nouvelle subjectivité par l'intégration des idéaux normatifs dominants. Ceux-ci élèvent la figure anthropologique de l'« homme-performant», préfigurant le modèle des sujets qui travaillent à leur propre autoréification. Dans un essai socioéconomique remarquable, " $L a$ nouvelle raison du monde-Essai sur la société néolibérale » Dardot et Laval [2] étudient les effets des valeurs du néolibéralisme sur la subjectivité contemporaine. Fondés sur le modèle entrepreneurial, les auteurs remarquent comment le discours «psy » rejoint le discours économique : des méthodes psy ou des techniques de coaching, " prouvées et efficaces », promettent la construction de soi, associant la maîtrise de ses potentialités individuelles à la meilleure jouissance de son corps. Cela produit une intégration des nouvelles normes économiques assurant le lien social au niveau des individus. Les sujets doivent ainsi s'autogérer selon l'idéal de gestion de l'entreprise de soi, dans un univers cruellement compétitif.
Il s'agirait de la promotion d'un nouvel idéal de l'homme, sans quoi il n'aurait jamais d'adhésion spontanée à ce modèle dans le circuit de production et de consommation de biens et de services. L'homme serait essentiellement soumis à la règle du profit maximal de son « capital humain »? Il adhère ainsi, inconsciemment, à un «modèle de performance/jouissance ». Le processus de normalisation serait assuré par des techniques disciplinaires qui vont constituer son "dispositifd'efficacité». Au niveau des organisations, la cohésion standardisée est fournie par l'usage massif de la communication qui donne les contours de la représentation des normes de l'individu. Autrement dit, le tissu culturel participe de la stratégie d'asservissement volontaire à la standardisation des normes de la société. Cette normalisation subjective singulière est indissociablement liée au modèle de gestion de ressources dans l'entreprise : élaboration des projets, codification des procédures, et contrôle de réalisation d'objectifs. L'évaluation serait inhérente à ce processus, puisqu'elle est supposée permettre de vérifier l'adhésion de l'individu à la norme de conduite attendue et de réguler son implication subjective.

L'évaluation, contrairement à sa présentation administrative objective, est une technique qui produit des effets subjectifs dans un champ concurrentiel. Elle ne vise pas tout à fait l'adaptation et l'intégration de l'individu, mais surtout l'intensification de ses performances. Cela doit pousser l'individu à se conformer au modèle qui lui garantira sa réussite dans la compétition dans laquelle il doit se sentir engagé.

\section{Conclusion}

Nous comprenons mieux, dans ce contexte de civilisation, la « niche écologique » dans laquelle le questionnement sur l'efficacité comparée des techniques psychothérapiques développe son expansion. C'est par la prise de conscience de ces enjeux et en se désengageant de l'emprise néfaste du «pur quantitatif » que l'on pourra accéder à connaître la réelle qualité des pratiques de soins, dans toute leur complexité.

Le paradigme des recherches, selon les principes de l'EBM, est certainement dépassé dans notre champ d'étude. Les cliniciens de bon sens ne peuvent qu'éprouver un profond désenchantement face aux incantations pseudoscientifiques que cette transposition a suscitées. Cette attente illusoire des preuves d'efficacité sur une base scientiste, c'est-à-dire purement technophile, entre des approches dont les fondements et les indications sont radicalement différents, comporte en elle-même une imposture au sein de laquelle toute rhétorique quantitative devrait être prise pour une avancée de la science véritable. En revanche, des recherches faites sur des bases méthodologiques, tenant compte de la spécificité des changements psychiques évalués, permettent de prouver, de manière empirique, tout l'intérêt des approches psychodynamiques quand les indications sont correctement posées. 
Face à des logiques évaluatives, fondées sur la base d'un savoir dominé par un paradigme, où le quantitatif devient le seul critère de vérité et la naturalisation du psychisme, la seule voie de légitimation scientifique, il est important d'affirmer que la validation des approches psychothérapeutiques, notamment psychanalytiques, requiert une stratégie complexe et diversifiée. Celle-ci pourra, certes, comporter une pluralité de méthodes de recherche, mais devra, au premier plan, réinstaurer la reconnaissance insubstituable du savoir qualitatif et de la valeur du singulier. En effet, tout programme de recherche psychothérapique, afin de démontrer son pouvoir de refléter la réalité de la clinique, nécessite d'intégrer la notion d'hypercomplexité propre à l'intersubjectivité et doit se rapprocher de la pratique réelle des praticiens. Enfin, aucun résultat des recherches expérimentales ne pourra prescrire une clinique standardisée se substituant à la créativité des cliniciens agissants dans le libre arbitre de leur interactivité authentique, dans la plupart des cas, partiellement indéterminé. Et pour cela même, dans une démarche décisionnelle liée à une politique de soins, il est primordial de se garder de toute transposition hâtive des résultats de la recherche fondamentale aux applications impliquant la gestion étatique de notre discipline.

\section{Bibliographie}

1. Briffault X, Lamboy B (2008) Les psychothérapies en France. Données quantitatives. In: Champion F (ed) Psychothérapie et société, Armand Colin, Paris
2. Dardot P, Laval C (2009) « La nouvelle raison du monde-Essai sur la société néolibérale » La Découverte, Paris

3. Fischman G (ed) (2009) L'évaluation des psychothérapies et de la psychanalyse. Fondements et enjeux. Contributions de Advenier F, Baruch C, Brusset B, Chartier JP, de Rotten Y, Despland JN, Duruz N, Falissard B, Gori R, Grosbois P, Guelfi JD, Laurent E, Ogien A, Perron R, Thurin JM, Thurin M, Widlöcher D Masson, Paris,

4. Inserm (2004) Psychothérapies : trois approches évaluées. Expertise collective, (Canceil O, Cottraux J, Falissard B, Flament M, Miermont J, Swendsen J, Teherani M, Thurin JM Paris

5. Kazdin A (1981) Drawing valid inferences from case studies. J Consult Clin Psychol 49:183-92 (In: Kazdin A (2003) Methodological Issues \& Strategies in Clinical Research. Amer Psychol Assoc Washington)

6. Lambert M, Bergin A, Garfield S (2004) Introduction and historical overview. In: Bergin \& Garfield's Handbook of psychotherapy and behavior change. Ed John Wiley, New York, pp. 3-15

7. Laurent E (2008) Lost in cognition. Éditions Cécile Defaut, Paris

8. Le Blanc G (2004) L'inévaluable-Actualité de Canguilhem. La Cause freudienne 57:115-22

9. Leuzinger-Bohleber M, Sthur U, Rüger B, Beutel M (2002) How to study the quality of psychoanalytic treatments and their longterm effects on patients' well-being: a representative, multiperspective follow-up study. Int J Psychoanal 84:263-90

10. Lopez A (2009) L'évaluation un enjeu de pouvoir à dépasser. In: Mesurer...les évaluations Rhizome 34 www.orspere.fr 2-3

11. Westen D, Novotny C, Thompson-Brenner H (2004) The empirical status of empirically supported psychotherapies: assumptions, findings, and reporting in controlled clinical trials. Psychol Bull 4:631-63 (Repr in Psychodynamic Diagnostic Manuel Alliance of Psychoanalytic Organisations, Silver Spring 2006)

12. Widlöcher D, Marie-Cardine M, Braconnier A, Hanin B (2006) Choisir sa psychothérapie. Les écoles, les méthodes, les traitements. Odile Jacob, Paris

13. Zarka YC (2009) L'évaluation : un pouvoir supposé savoir. Cités $37: 113-23$ 